\title{
DEMONSTRATION OF TRANSVERSE-TO-LONGITUDINAL EMITTANCE EXCHANGE AT THE FERMILAB PHOTOINJECTOR *
}

\author{
A. Johnson ${ }^{1}$, J. Ruan ${ }^{1}$, H. Edwards ${ }^{1}$, T. Koeth ${ }^{1}$, A. Lumpkin ${ }^{1}$, P. Piot ${ }^{2,3}$, J. Santucci ${ }^{1}$, \\ Y.-E Sun ${ }^{2}$, R. Thurman-Keup ${ }^{1}$ \\ ${ }^{1}$ Accelerator Division, Fermi National Accelerator Laboratory, Batavia, IL 60510, USA \\ 2 Accelerator Physics Center, Fermi National Accelerator Laboratory, Batavia, IL 60510, USA \\ ${ }^{3}$ Department of Physics, Northern Illinois University, DeKalb, IL 60115
}

\begin{abstract}
Phase space manipulation techniques within two degrees of freedom are foreseen to enhance the performances of next generation accelerators such as high-energy physics colliders and accelerator based light sources. At the Fermilab A0 photoinjector, a proof-of-principle experiment to demonstrate the exchange of the transverse and longitudinal emittances is ongoing. The emittance exchange beamline consists of a $3.9 \mathrm{GHz}$ normal conducting deflecting mode cavity flanked by two doglegs. Electron bunches with charges of $250 \mathrm{pC}$ and energy of $14.3 \mathrm{MeV}$ are routinely sent through the exchanger. In this paper, we report our latest results on the demonstration of emittance exchange obtained with significantly improved beam diagnostics. We also compare our experimental results with a simple numerical model.
\end{abstract}

\section{INTRODUCTION}

The emittance exchange (EEX) experiment can be described through linear optics as a transformation matrix, $M_{\mathrm{EEX}}$, that operates on the initial horizontal transverse emittance parameters $\left(x, x^{\prime}\right)$ and longitudinal emittance parameters $\left(z, \frac{\Delta p}{p}\right)$, leaving the vertical transverse emitance unchanged [1, 2, 3]:

$$
\left(\begin{array}{c}
x \\
x^{\prime} \\
z \\
\frac{\Delta p}{p}
\end{array}\right)_{\text {out }}=\left(\begin{array}{cc}
A & B \\
C & D
\end{array}\right)\left(\begin{array}{c}
x \\
x^{\prime} \\
z \\
\frac{\Delta p}{p}
\end{array}\right)_{\text {in }}
$$

where $\boldsymbol{A}, \boldsymbol{B}, \boldsymbol{C}$ and $\boldsymbol{D}$ are $2 \times 2$ sub-matricies of $M_{\mathrm{EEX}}$. A perfect emittance exchange matrix will exist when the elements of the diagonal sub-blocks, $\boldsymbol{A}$ and $\boldsymbol{D}$ become zero and the $\boldsymbol{B}$ and $\boldsymbol{C}$ sub-blocks are populated. Due to the finite length of our $T M_{110}$ deflecting mode cavity, many of the on-diagonal elements are non-zero [4]. Higher-order effects such as space charge and coherent synchrotron radiation also contribute to imperfect exhange.

\section{EXPERIMENTAL SETUP}

The EEX experiment is conducted at Fermilab's A0 Photoinjector [7]; see Figure 1. The three solenoid lenses that

\footnotetext{
* This work was supported by Fermi Research Alliance, LLC under contract No. DE-AC02-07CH11359 with the U.S. Department of Energy.
}

surround the gun control the beam's transverse size and emittance. The beam then enters a $1.3 \mathrm{GHz}$ superconducting radio frequency booster cavity bringing the final energy to $14.3 \mathrm{MeV}$. Following this final acceleration stage are various diagnostic crosses consisting of either optical transition radiation (OTR) or cerium doped yttrium aluminum garnet (YAG:Ce) crystal viewers. Also found in the beamline are quadrupoles, dipole correctors and beam position monitors.

The EEX beamline at the A0 Photoinjector consists of a liquid nitrogen cooled, normal conducting $3.9 \mathrm{GHz}$ $T M_{110}$ deflecting mode cavity placed between two magnetic doglegs $[5,6]$. The doglegs each generate a bending angle of $\alpha=22.5^{\circ}$, a horizontal dispersion of $D=0.33 \mathrm{~m}$. The drift length betweeen dipoles forming each dogleg is $L$. The longitudinal electric field of the $T M_{110}$ cavity is zero on axis and grows linearly off axis, while the vertical magnetic field produces a time-dependent transverse kick with respect to the synchronous particle. Assuming the thin lens approximation of the EEX beamline elements generates a perfect EEX matrix. When the deflecting mode cavity strength is defined to be $-1 / D$, the diagonal sub-blocks become zero:

$$
M_{\mathrm{EEX}}=\left(\begin{array}{cccc}
0 & 0 & -\frac{1}{\alpha}-\frac{L}{D} & -\alpha L \\
0 & 0 & -\frac{1}{D} & -\alpha \\
-\alpha & -\alpha L & 0 & 0 \\
-\frac{1}{D} & -\frac{1}{\alpha}-\frac{L}{D} & 0 & 0
\end{array}\right)
$$

\section{EXPERIMENTAL METHODS}

Transverse emittance measurements are made using the slit measurement technique [8]. Transverse beam profiles are measured by inserting an OTR screen at X3 and X23. Divergence measurements are made by inserting a tungsten multi-slit mask into the beam path at both X3 and X23. The masks consist of $50 \mu \mathrm{m}$ wide slits separated by $1 \mathrm{~mm}$, except at X23 where the horizontal slits are separated by $4 \mathrm{~mm}$. Figure 2 shows a typical set of incoming horizontal beam size data and a complete set of horizontal beamlets as viewed on the X6 YAG:Ce screen. Divergence calculations use the average of the Gaussian fits of the slit images and divide by the drift distance from X3 to X6. For comparison, Figure 3 shows the transverse beam profile after exchange at X23 and the beamlets as viewed at X24. The average beam size and divergence values are shown in Table 1. 


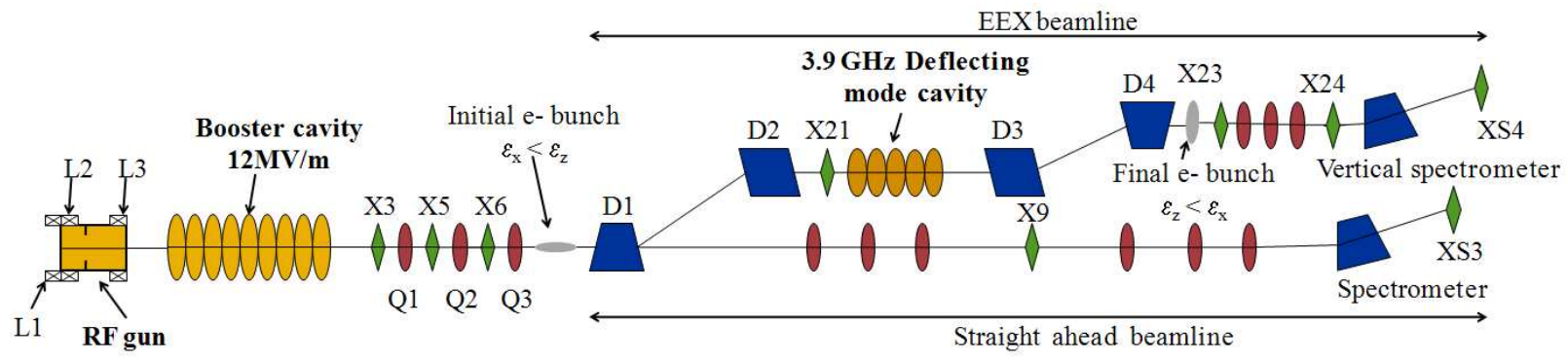

Figure 1: Top view of the A0 photoinjector showing elements pertinent to performing emittance exchange. Elements labeled "X" are diagnostics stations (beam viewers and/or multi-slit mask locations), "L" are solenoid lenses, and "Q" are quadrupole magnets.

Table 1: Transverse beam size, $\sigma_{i}(i=x, y)$, in $\mathrm{mm}$ as measured at X3 (in) and X23 (out) and divergence, $\sigma_{i}^{\prime}$ $(i=x, y)$, in mrad as measured at X6 (in) and $\mathrm{X} 24$ (out). Uncertainty in Gaussian fit of the peaks(s) is represented by the trailing parenthetical number.

\begin{tabular}{ccccc}
\hline \hline & $x_{\text {in }}$ & $y_{\text {in }}$ & $x_{\text {out }}$ & $y_{\text {out }}$ \\
\hline$\sigma_{i}$ & $1.589(1)$ & $2.166(1)$ & $4.215(3)$ & $1.798(1)$ \\
$\sigma_{i}^{\prime}$ & $0.081(3)$ & $0.053(2)$ & $0.113(1)$ & $0.090(7)$ \\
\hline \hline
\end{tabular}

Longitudinal emittance is derived from bunch length and energy spread measurements. Installed in the beam enclosure is a Hamamatsu C5680 streak camera which can be used to measure the electron bunch length both before and after the exchange at the picosecond level [9]. Sub-picosecond bunch length measurements use a MartinPupplett interferometer that is located at X24 of the EEX beamline [10]. A spectrometer magnet and viewing screen are located at the end of each beamline to measure the central momentum and momentum spread of the beam. The spectrometer magnet at the end of the EEX line is vertical to avoid residual dispersion from the exchanger.

\section{SIMULATION}

A linear transfer matrix model of the EEX beamline has been assembled within Matlab in an effort to explore the behavior of the EEX line. It includes the quadrupole and dipole magnets, and has a hybrid thin/thick lens model of the deflecting mode cavity which agrees well with the measured cavity transfer function [11]. The simulation also includes linear space charge forces (both transverse and longitudinal) adapted from the Trace3D user manual [12]. The space charge effect through the dipoles is achieved by slicing the dipoles longitudinally, and interleaving space charge defocusing between slices. The simulation requires the phase space parameters on input to the beamline at X3, which is just downstream of the booster cavity and is where the incoming transverse emittance measurements are usually made. The simulation is designed to be used online in the sense that the magnet settings are read from the actual settings, however it can also be used offline where the settings are input by the user. Equations 3 and 4 show the measured $\left(M_{\text {Data }}\right)$ [13] and linear model transfer $\left(M_{\mathrm{LMT}}\right)$ matrices of the EEX beamline from X3 to X23.

$$
\begin{aligned}
& M_{\text {Data }}=\left(\begin{array}{rrrr}
0.02 & -0.23 & 4.75 & 0.40 \\
-0.02 & 0.11 & -0.02 & 0.21 \\
0.23 & 0.63 & -0.21 & 0.00 \\
-0.09 & 4.89 & 0.13 & 0.08
\end{array}\right) \\
& M_{\text {LMT }}=\left(\begin{array}{rrrr}
0.01 & 0.01 & 5.20 & 0.33 \\
0.01 & 0.01 & -0.07 & 0.19 \\
0.19 & 0.67 & 0.03 & 0.00 \\
-0.07 & 5.06 & 0.28 & 0.03
\end{array}\right)
\end{aligned}
$$

\section{EEX RESULTS}

In Table 2, we present the results of our most recent EEX measurement and compare with simulation. The measurement was taken with an electron bunch charge of $250 \mathrm{pC}$ at $14.3 \mathrm{MeV}$. Incoming transverse phase space was adjusted using the three input quadrupole magnets to yield a minimum output bunch length and energy spread product. However, according to our simulation, the optimized input quadrupole settings for minimum longitudinal emittance will lead to a beam with a time-energy correlation, which our current diagnostic system cannot measure. In addition, the EEX matrix as measured does not yield an ideal exchange matrix. In particular, the R43 element is non-zero simply due to the cavity being finite in length.

\section{CONCLUSION}

A proof-of-principle EEX experiment utilizing the Fermilab A0 Photoinjector has been completed for a beam charge of $250 \mathrm{pC}$. We are currently limited in our measurement of longitudinal phase space by the present diagnostic system's inability to account for time-energy correlation. Investigations into the effects of CSR and space charge are ongoing. Ugrades to the beamline include the installation 

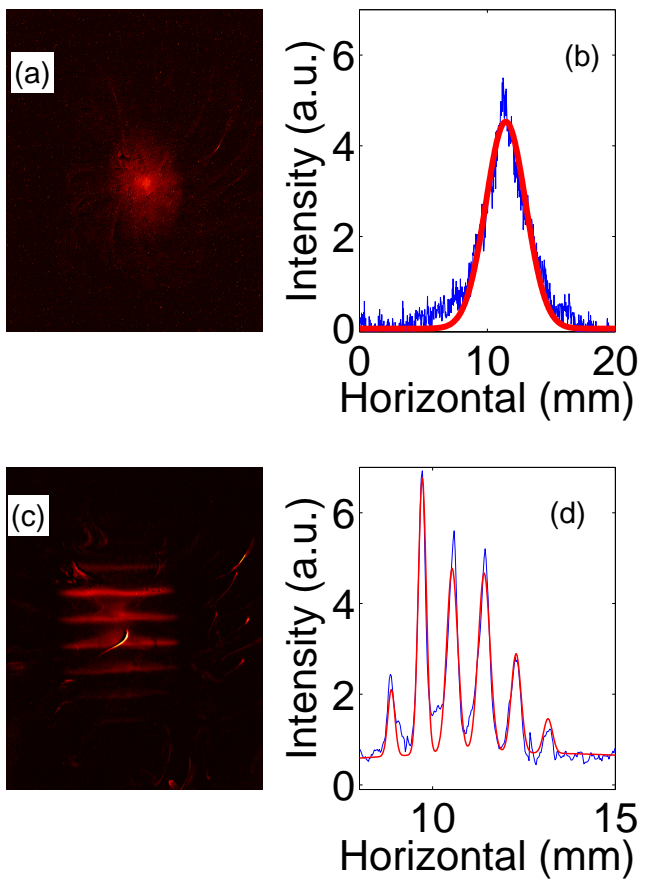

Figure 2: Incoming transverse emittance images. Beam profile image taken at X3 OTR screen (a) with Gaussian fits to the $x$-projection (b) and the slit images taken on a YAG:Ce crystal screen located at X6 (c) with respective Gaussian fits (d). Images are rotated $90^{\circ}$.

Table 2: Comparison of direct measurements of horizontal transverse $(x)$ to longitudinal $(z)$ emittance exchange to simulation. Emittance measurements are shown in mmmrad.

\begin{tabular}{rrrrr}
\hline \hline & \multicolumn{2}{c}{ Simulated } & \multicolumn{2}{c}{ Measured } \\
& \multicolumn{1}{c}{ In } & Out & \multicolumn{1}{c}{ In } & \multicolumn{1}{c}{ Out } \\
\hline$\epsilon_{\mathrm{x}}$ & 3.7 & 18.7 & $3.7 \pm 0.1$ & $13.9 \pm 1.2$ \\
$\epsilon_{\mathrm{y}}$ & 3.3 & 3.3 & $3.3 \pm 0.1$ & $4.7 \pm 0.4$ \\
$\epsilon_{\mathrm{z}}$ & 16.2 & 9.2 & $16.2 \pm 1.5$ & $7.7 \pm 2.0$ \\
\hline \hline
\end{tabular}

of YAG:Ce screens at all diagnostic cross locations, which will allow for a reduction of the bunch charge thereby lessening space charge effects.

\section{REFERENCES}

[1] M. Cornachia, and P. Emma, Phys. Rev. ST Accel. Beams 6 030702 (2003).

[2] P. Emma, Z. Huang, K.-J. Kim, and P. Piot, Phys. Rev. ST Accel. Beams 9, 100702 (2006).

[3] K.-J. Kim and A. Sessler, AIP Conf. Proc. 821, 115 (2006).

[4] D.A. Edwards, private communication.
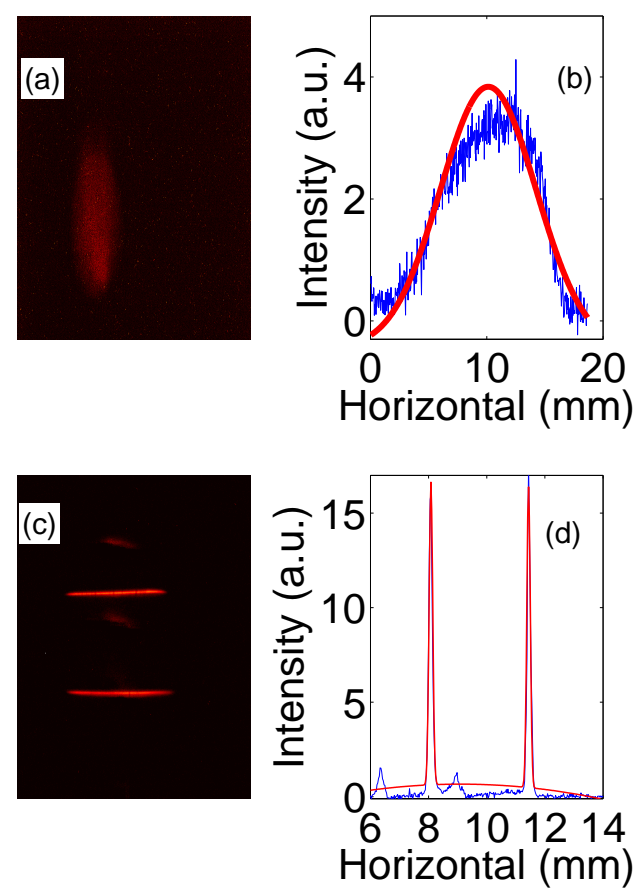

Figure 3: Outgoing transverse emittance images. Beam profile image taken at X23 OTR screen (a) with Gaussian fits to the $x$-projection (b) and the slit images taken on a YAG:Ce crystal screen located at X24 (c) with respective Gaussian fits (d). Images are rotated $90^{\circ}$.

[5] T. W. Koeth, et al, Proceedings of the 2008 International Linac Conference (LINAC08), Victoria, BC, 667 (2009).

[6] T. W. Koeth, et al, Proceedings of the 2009 Particle Accelerator Conference (PAC09), Vancouver, BC, FR5PFP020 (2009).

[7] J.-P. Carneiro, et al, Phys. Rec. ST Accel. Beams 8, 040101 (2005).

[8] C. H. Wang, et al, "Slits Measurement of Emittance on TTF," International Conference on Accelerator and Large Experimental Physics Control Systems, Trieste, Italy (1999).

[9] A. H. Lumpkin, et al, "Initial Synchroscan Streak Camera Imaging at the A0 Photoinjector," Proceedings of the 2008 Beam Instrumentation Workshop, Lake Tahoe, CA (2008).

[10] R. M. Thurman-Keup, et al, "Bunch Length Measurement at the Fermilab A0 Photoinjector using a Martin-Pupplett Interferometer," Proceedings of the 2008 Beam Instrumentation Workshop, Lake Tahoe, CA (2008).

[11] E. Branlard, "Study of a 5 Cell Copper Cavity at $3.9 \mathrm{GHz}$ ", Unpublished (2007).

[12] Trace3D, http://laacg1.lanl.gov/laacg/services/traceman.pdf

[13] T. W. Koeth, "An Observation of a Transverse to Longitudinal Emittance Exchange at the Fermilab AO Photoinjector" (Doctoral Dissertation), Rutgers University, Piscataway, NJ. (2009). 